\title{
MÉTODOS PREVENTIVOS DE DESEQUILÍBRIOS METABÓLICOS EM BOVINOS - HIPOCALCEMIA E CETOSE: REVISÃO DE LITERATURA
}

\author{
FACCO, Fabíola Secretti ${ }^{1}$ \\ SILVA, Marlon Richard Hilário da ${ }^{2}$ \\ VRISMAN, Dayane Priscila ${ }^{3}$ \\ STRUCHER, Felipe ${ }^{3}$ \\ COSTA, Fernando Corazza ${ }^{3}$
}

RESUMO: Com a intensificação da produção, os distúrbios metabólicos estão cada vez mais frequentes, e a presença destes distúrbios agrega custos á produção, além de comprometer a lactação atual destes animais. A ocorrência de tais distúrbios pode ser evitada, se durante o pré-parto forem adotados manejos nutricionais visando à prevenção destas enfermidades. A hipocalcemia e a cetose estão entre os distúrbios metabólicos de maior ocorrência em rebanhos de alta produção, devido ao intenso requerimento nutricional, oscilações hormonais e severo balanço energético negativo, portanto, é importante conhecer como estas enfermidades ocorrem, para que sejam adotados no pré-parto medidas adequadas, como manejo alimentar, adequação da condição corporal e utilização de dietas aniônicas, objetivando à prevenção das mesmas.

Palavras-chave: Cetose. Dieta aniônica. Distúrbios metabólicos. Hipocalcemia. Ionóforos

\section{PREVENTIVE METHODS OF METABOLIC IMBALANCES IN BOVINE - HYPOCALCEMIA AND KETOSIS: LITERATURE REVIEW}

\begin{abstract}
SUMMARY: With the intensification of productivity, metabolic disorders are increasingly frequent, and the presence of these disorders adds to the production costs and compromise the current lactation these animals. The occurrence of such disturbances can be avoided during the pre-calving nutritional management are adopted for the prevention of these diseases. The hypocalcemia and ketosis are among the most frequent metabolic disorders in high producing herds, due to the intense nutritional needs, hormonal fluctuations and severe negative energy balance, so it is important to know how these diseases occur, to be adopted in the antepartum appropriate measures such as feed management, adequacy of body condition and use of anionic diets, aiming at prevention.
\end{abstract}

Keywords: Ketosis. Anionic diet. Metabolic disorders. Hypocalcemia. Ionophores.

\section{INTRODUÇÃO}

Mudanças significativas têm sido verificadas nos últimos anos no tocante a alimentação dos ruminantes, com o intuito de incrementar melhoras sobre a sanidade e produtividade, porém, juntamente com estas melhoras surgem desequilíbrios metabólicos e digestivos, os quais estão ocorrendo com maior frequência (CHURCH, 1993; NAGARAJA; TITGEMEYER, 2007).

\footnotetext{
${ }^{1}$ Departamento de Medicina Veterinária, Universidade Estadual do Centro-Oeste UNICENTRO, Guarapuava, Paraná, Brasil. Endereço para correspondência: CEP: 85340-000, Rio Bonito do Iguaçú, PR, email: fabiolafacco@hotmail.com.

${ }^{2}$ Médico Veterinário, mestre em Produção animal e Pastagens, professor do Curso de Medicina Veterinária da Universidade Estadual do Centro-Oeste (UNICENTRO), Guarapuava, PR - Brasil, e-mail: marlon_rhs@hotmail.com.

${ }_{3}^{3}$ Departamento de Medicina Veterinária, Universidade Estadual do Centro-Oeste UNICENTRO, Guarapuava, Paraná, Brasil.
} 
O período de transição na vaca leiteira é caracterizado por mudanças metabólicas, este se inicia nas três últimas semanas de gestação e se estende até a terceira semana de lactação (TEDESCO et al., 2004). O período seco deve permitir a regeneração das células epiteliais desgastadas, formação de colostro de qualidade e assegurar um bom desenvolvimento do feto, bem como ajuste das reservas corporais. Além dos transtornos metabólicos, ocorrem alterações endócrinas e imunológicas, assim como fatores relacionados ao estresse do ambiente em que as vacas são submetidas entre o período seco e a lactação (NETO et al., 2011). Neste período as demandas energéticas são maiores devido ao crescimento fetal e lactogênese (WHATHES et al., 2007), podendo resultar em importantes perdas econômicas decorrente dos desequilíbrios nutricionais que afeta também o desempenho produtivo e reprodutivo dos animais (HAYIRLI et al., 2002).

A ocorrência de problemas metabólicos em vacas agrega valores significativos ao produtor, devido aos custos de tratamento, diminuição de produção e fertilidade (KUNKEL, 2007). Além destes, existem custos com doenças correlacionados as patologias metabólicas. Dentre estas doenças, destaca-se, a hipocalcemia clínica que aumenta o risco da vaca desenvolver mamite (GOFF; HORST, 1997a), assim como cetose subclínica, a qual predispõe o desenvolvimento de um deslocamento de abomaso (LE BLANC et al., 2005).

Devido à alta prevalência de distúrbios metabólicos na bovinocultura de leite e as percas econômicas que envolvem estas doenças, o presente trabalho propõem uma revisão bibliográfica sobre Hipocalcemia e Cetose, focando principalmente na etiologia e métodos preventivos de tais enfermidades.

\section{HIPOCALCEMIA}

A hipocalcemia é também conhecida como paresia do puerpério, febre do leite ou febre vitular e manifesta-se geralmente nas primeiras 12 a 24 horas após o parto (SANTOS, 2006). Sua ocorrência é decorrente do aumento da exigência de cálcio $(\mathrm{Ca})$ por ocasião da síntese de colostro, além da demanda para crescimento e mineralização do tecido ósseo fetal, aumentando a necessidade do deslocamento de Ca da mãe para o feto no pré-parto (RODRIGUES, 2004).

Segundo Santos (2006) à alta demanda de Ca nos dias que precedem o parto e as primeiras semanas pós-parto causam diferentes graus de hipocalcemia, esse fato ocorre devido à queda dos níveis sanguíneos de $\mathrm{Ca}$ e $\mathrm{Ca}$ ionizado $\left(\mathrm{Ca}^{++}\right)$. Essa alteração nos níveis de $\mathrm{Ca}$ pode ou não apresentar sintomatologia clínica, sendo que a hipocalcemia clínica ocorre em $3 \%$ a $15 \%$ das vacas leiteiras anualmente, enquanto $50 \%$ das vacas desenvolvem hipocalcemia subclínica, sendo esta a que causa maiores danos e prejuízos a bovinocultura de leite. 
Os animais que apresentam hipocalcemia subclínica consomem menores quantidades de matéria seca (MS) (HORST et al., 1997) e ficam mais susceptíveis a desenvolverem síndrome da vaca caída, retenção de placenta, prolapso uterino, deslocamento de abomaso, baixo desempenho reprodutivo e maior incidência de mamite (BEEDE, 1992; OETZEL, 1995).

De acordo com Gonzáles e Silva (2006), o Ca sérico em bovinos é mantido entre 8,8 a 10,4 miligramas/decilitro (mg/dL) através da homeostase. Já Santos (2006) cita valores normais de Ca entre 8,5 a 11,5 mg/dL, e $\mathrm{Ca}^{++}$entre 4,5 a 5,5 mg/dL, esse valor equivale a 6 gramas $(\mathrm{g}) \mathrm{de}$ Ca no organismo de uma vaca de 600 kilogramas (kg) (GOFF, 2000). As reservas e a mobilização de Ca prontamente disponível estão reduzidas ao final da gestação, dificultando a manutenção dos níveis normais no início da lactação (RADOSTITS, 2002).

De acordo com Santos (2006) ao final da gestação, uma vaca de $650 \mathrm{~kg}$ necessita de $20 \mathrm{~g}$ de Ca absorvível, sendo que no primeiro dia de lactação esse requerimento de Ca se eleva para 50 a 60 g. A concentração de Ca em um litro de leite é de 1,1 a 1,2 g, porém, no colostro esse valor é de 2,0 a 2,3 g. Assim, é necessário de 7 a 10 vezes a quantidade de Ca sérica para repor o valor necessário para a produção de $10 \mathrm{~kg}$ de colostro na primeira ordenha.

A deficiência na homeostase do Ca sanguíneo esta relacionada com a sensitividade das células ao Paratormônio (PTH). Vacas que ao final da gestação recebem dietas com Ca superior aos requerimentos limitam a secreção de PTH e, no parto o mecanismo de reabsorção óssea e de aumento da absorção intestinal de Ca estarão hipofuncionais (SANTOS, 2006).

Ao final da gestação, medidas adequadas no manejo e alterações na dieta, podem reduzir a níveis mínimos a ocorrência deste distúrbio (LEAN; DEGARIS, 2009).

\section{Homeostase do Cálcio}

Para a manutenção dos níveis sanguíneos de $\mathrm{Ca}$, diferentes mecanismos podem ser ativados frente a uma demanda mineral, objetivando manter sua concentração dentro dos níveis ideais (DUKES, 1996). Os níveis normais de Ca no sangue dependem do consumo e absorção intestinal de $\mathrm{Ca}$, da reabsorção do $\mathrm{Ca}$ do tecido ósseo e do filtrado glomerular. Estes níveis são controlados pelo PTH, pela Calcitonina e Vitamina $\mathrm{D}_{3}$ (JONES et al., 2000; GONZÁLES; SILVA, 2006; SANTOS, 2006).

O PTH é produzido nas glândulas paratireóides, têm como órgãos alvo os rins e os ossos, a ação deste eleva os níveis de Ca e diminui o de fósforo $(\mathrm{P})$ nos líquidos extracelulares. Nos ossos ele inibe a síntese de colágeno nos osteoblastos, aumenta a desmineralização pelos osteócitos, aumenta a osteólise pelos osteoclastos e aumenta a taxa de maturação celular das células precursoras de osteoblastos e osteoclastos. Nos túbulos renais o PTH aumenta a adenosina monofosfato cíclico favorecendo a reabsorção de Ca e desfavorecendo a reabsorção de P. Quando 
o $\mathrm{Ca}^{++}$está aumentado a paratireóide, através do feedback negativo, diminui a secreção de PTH, e quando está baixo, ela aumenta a secreção (CUNNINGHAM, 1993).

A regulação da vitamina $\mathrm{D}_{3}$ se da por mecanismos que envolvem Ca, P e PTH. Com o aumento do PTH, há um aumento na formação renal de vitamina $\mathrm{D}_{3}$, que devera estimular a absorção de Ca e P no intestino, nos ossos ela estimula a osteólise e nos rins atua diminuindo a excreção renal do Ca e P (CUNNINGHAM, 1993).

A calcitonina é produzida pelas células $\mathrm{C}$ presentes na tireóide, paratireóide e timo. Com ação principalmente nos ossos, ela inibe a desmineralização óssea, e sua ação nos rins é diminuir a reabsorção de Ca e P nos túbulos renais (CUNNINGHAM, 1993). Sua regulação é feita pelo feedback positivo, assim, o aumento na concentração $\mathrm{Ca}^{++}$estimula a síntese e liberação de calcitonina (SANTOS, 2006).

\section{Métodos Preventivos}

As medidas preventivas têm por objetivo diminuir a intensidade e a duração da hipocalcemia fisiológica (TEIXEIRA et al., 2003). Esta consiste na aplicação de um bom manejo nutricional no pré-parto, com dietas balanceadas e nutritivas (RODRIGUES, 2004).

A prevenção da hipocalcemia baseia-se praticamente no uso de dietas deficientes em Ca, que estimulará a secreção e atividade do PTH, e na manipulação do balanço cátio-aniônico (BCA) da dieta, influenciando a atividade do PTH e, portanto, o mecanismo de absorção ativa de Ca. A utilização do BCA no pré-parto pode influenciar a incidência de hipocalcemia, dietas altas em cátions, especialmente $\mathrm{Na}$ e $\mathrm{K}$, tendem a induzir este distúrbio metabólico, e altos níveis de ânions, principalmente $\mathrm{Cl}$ e S, podem prevenir a doença (SANTOS, 2006).

Segundo Obaa (2011) a concentração elevada de cálcio na dieta não é a principal causa para a ocorrência da hipocalcemia no parto, mas sim a elevada diferença entre cátions e anions na dieta.

\section{Dietas com baixa concentração de cálcio}

Utilizando-se dietas com baixas concentrações de $\mathrm{Ca}$ no período pré-parto, a níveis menores de 20g/dia de Ca absorvível (SANTOS, 2006; THILSING-HANSEN et al., 2002; OBAA et al., 2011), ou abaixo de 30 g/dia (GOFF, 2000) diminui-se a ocorrência de hipocalcemia.

O NRC (2001) recomenda o fornecimento de baixos níveis de Ca no pré-parto, com menos de $15 \mathrm{~g} \mathrm{de} \mathrm{Ca} / \mathrm{vaca} / \mathrm{dia}$, visando estimular os mecanismos que atuam na liberação do $\mathrm{Ca}$ dos ossos para o sangue e manter seus níveis próximos à normalidade em períodos de grande 
exigência, através da redução da calcitonina e do aumento do PTH e colecalciferol (vitamina D não ativa).

Dietas com baixas quantidades de $\mathrm{Ca}$ e $\mathrm{P}$ durante o período pré-parto, elevam as concentrações sanguíneas de Vitamina D3 e PTH. Com isso o nível sanguíneo deste hormônio estará alto quando a demanda de Ca aumentar, alterando os mecanismos de reabsorção óssea por ativação dos osteoclastos (CAVALIERI; SANTOS, 2000).

Horst et al. (1997) recomenda dietas para vacas no pré-parto com 15-20 g de Ca por dia, alimentadas durante os últimos 14 dias de gestação, seguida de dieta pós-parto com alta inclusão de Ca. Com esta alimentação os osteoclastos são ativados e estes estimulam a reabsorção óssea e a retenção de Ca nos túbulos renais. Santos (2006) relatou que além da reabsorção óssea, a absorção intestinal de Ca também é ativada através da síntese da vitamina $\mathrm{D}$, que é estimulada pelo PTH.

Embora esta seja uma maneira eficaz de reduzir a ocorrência do distúrbio clínico, muitas vezes não se consegue sucesso total com esta prática, pois a incidência do fenômeno subclínico continua a afetar negativamente o rebanho. Estudos têm demonstrado que o principal fator que afeta os níveis séricos de Ca e a capacidade do organismo de se ajustar a sua demanda é o BCA da vaca nesse período, o qual é pouco influenciado pelos níveis de Ca na dieta (GOFF; HORST, 1997a).

\section{Dietas aniônicas}

Os eletrólitos da dieta são classificados como ânions ou cátions. Os ânions possuem carga negativa, sendo o cloro $(\mathrm{Cl})$, enxofre $(\mathrm{S})$ e $\mathrm{P}$ os mais importantes. Já os cátions apresentam carga positiva e os mais importantes são o sódio $(\mathrm{Na})$, potássio $(\mathrm{K}), \mathrm{Ca}$, magnésio $(\mathrm{Mg}) . \mathrm{O} \mathrm{Na}, \mathrm{K}$ e Cl, são denominados como "íons fortes" (CAVALIERI; SANTOS, 2000). Além destes, Santos (2006) classifica também o S como "íon forte". Eles recebem essa denominação pelo fato de possuírem uma maior taxa de absorção no trato digestório e maior concentração de cargas miliequivalentes (mEq).

O uso de sais aniônicos ou a manipulação do BCA da dieta tem sido utilizado como método efetivo na manutenção dos níveis de $\mathrm{Ca}$, prevenindo a hipocalcemia. O BCA é a relação entre a concentração, em mEq, de cátions e ânions nos fluidos corporais e devem se equalizar. Assim o influxo de íons corporais altera o balanço ácido-básico do organismo, e quando a quantidade de ânions é maior que a de cátions, o animal entra em acidose metabólica (SANTOS, 2006; HORST et al., 1997).

O corpo animal tem que manter sua neutralidade elétrica, tendo sempre equilíbrio entre cátions $\mathrm{H}^{+}, \mathrm{K}^{+}, \mathrm{Na}^{+}$e ânions $\mathrm{Cl}^{-}, \mathrm{SO}_{4}{ }^{-}{\mathrm{e} \mathrm{HCO}_{3}}^{-}$. Ao se fornecer uma dieta aniônica para vacas 
leiteiras no final da gestação, ocorre um aumento das concentrações intestinais de $\mathrm{Cl}^{-}$e $\mathrm{SO}_{4}{ }^{-}$. Estes ânions em maiores quantidades devem ser equilibrados com os cátions presentes no corpo do animal e aqueles que estão sendo absorvidos, aumentam-se a excreção de $\mathrm{HCO}_{3}{ }^{-}$da circulação para o lúmen intestinal, com isso ocorre uma leve queda no $\mathrm{pH}$ sanguíneo (CAVALIERI; SANTOS, 2000).

A acidose metabólica altera a responsividade dos tecidos ao PTH, reduzindo a refratividade do tecido ósseo e dos rins a este hormônio, resultando no aumento da taxa de reabsorção osteoclástica de $\mathrm{Ca}$ do tecido ósseo e aumento nos níveis séricos de vitamina D3 (SANTOS, 2006; HORST et al., 1997).

Cavalieri e Santos (2000) consideram uma dieta aniônica quando a diferença cátionaniôn calculada pela equação $[(\mathrm{Na}+\mathrm{K})-(\mathrm{Cl}+\mathrm{S})]$ fica entre -10 e $-20 \mathrm{mEq} / 100 \mathrm{~g}$ de $\mathrm{MS}$. Neste nível, provavelmente haverá acidose e redução na ocorrência de distúrbios metabólicos. Já Santos (2006) sugere dietas com BCA de - 50 a - $100 \mathrm{mEq} / \mathrm{kg}$ de MS para se ter melhor resposta no metabolismo de Ca e na prevenção de hipocalcemia. Para Davidson et al. (1995) e Moore et al. (2000), o valor do BCA da dieta deve ficar entre -100 e $-150 \mathrm{mEq} / \mathrm{kg}$ de MS para a manutenção da concentração plasmática de Ca dentro dos limites normais.

As concentrações de $\mathrm{Ca}$ na dieta devem ser mais altas em animais que recebem uma alimentação com diferença cátion-anion da dieta (DCAD) negativa, devido ao fato destas dietas causarem um aumento na excreção urinária de $\mathrm{Ca}$, e na reabsorção óssea. Vacas alimentadas com DCAD negativas tendo uma concentração de Ca na dieta de 9,9 ou 15 g/kg de MS ingerida, são ambas efetivas na prevenção da hipocalcemia, o que indica que pode não haver benefícios em alimentar vacas com concentrações de cálcio superiores a $10 \mathrm{~g} / \mathrm{kg}$ de MS, se a DCAD for negativa (OBAA et al., 2011).

Para formulações de dietas com sais aniônicos num primeiro momento é necessária a escolha de alimentos com baixos teores de Na e K (SANTOS, 2006). Segundo Goff e Horst (1997a) e Mulligan et al. (2006) a percentagem de K na dieta é mais importante do que a de Ca na prevenção da hipocalcemia. Segundo Rérat et al. (2009) existe um aumento na ingestão de MS e um efeito positivo no balanço de $\mathrm{Ca}$ e $\mathrm{P}$ em vacas de leite submetidas a uma alimentação no período pré-parto a base de forragem com baixas concentrações de K. Ramos-Nieves et al. (2009) relatam que vacas alimentadas com dietas aniônicas no pré-parto tem um aumento moderado na concentração de $\mathrm{Ca}_{\text {e } \mathrm{Ca}^{++}}$durante as primeiras 24 horas pós-parto.

Quando se utiliza ingredientes com teores baixos de $\mathrm{Na}$ e K, reduz-se a quantidade de sais aniônicos necessários para alterar a BCA da dieta para valores negativos, diminuindo assim riscos de alterar consumo de MS devido à palatabilidade (SANTOS, 2006). De maneira geral, silagem de milho, fenos de gramíneas e grãos possuem valores de DCAD mais baixos, enquanto 
pastagens diretas, silagens de pasto e fenos de leguminosas têm DCAD positiva (CORBELLINI, 1998).

Apesar de fosfatos serem ânions e reduzirem o BCA, a ingestão deste íon na dieta deve ser limitada em 40 a $50 \mathrm{~g} /$ dia pelo fato dele inibir a síntese de Vitamina D3 pelos rins e poder causar hipocalcemia (GOFF, 2000; SANTOS, 2006).

Os resultados da adição de sais aniônicos a dieta de vacas secas são observados em um curto espaço de tempo. Após 1 a 2 dias de inclusão na dieta, os sais aniônicos já irão reduzir o pH urinário e promover uma pequena acidose metabólica. É indicado que dietas acidogênicas sejam fornecidas por pelo menos uma a duas semanas antes do parto para que se obtenham melhores resultados (BEEDE, 1992; SANTOS, 2006).

\section{CETOSE}

A cetose é uma doença metabólica ocasionada pela queda na concentração de glicose e aumento excessivo de corpos cetônicos. É considerada o principal transtorno metabólico de vacas leiteiras de alta e média produção no inicio da lactação e tem sido associada com diminuição da produção, deficiência no desempenho reprodutivo e baixa imunidade (GRUMEER, 2008; BOBE et al., 2004).

Ocorre no inicio da lactação, geralmente entre os dias 8 e 60 do pós-parto, período em que o animal enfrenta um BEN (GONZÁLEZ; CAMPOS, 2003; DRACKLEY et al., 1992; GRUMMER, 1993), devido a alta demanda de energia e proteína para suprir as necessidades de manutenção e lactação, as quais não podem ser compensadas pela alimentação (JUCHEM et al., 2004; VAN KNEGSEL et al., 2007).

Há uma queda no consumo de alimento na última semana de gestação, permanecendo assim durante as primeiras cinco semanas da lactação (GOFF; HORST, 1997b). Nesta fase ocorre mobilização das reservas de gordura e aminoácidos para serem utilizados como fonte de energia através da lipólise e proteólise (GOFF; HORST, 1997b; JUCHEM et al., 2004; RUKKWAMSUK et al., 2005).

A cetose caracteriza-se pelo aumento na concentração de corpos cetônicos [acetoacetato (AcAc), $\beta$-hidroxibutirato (BHB) e acetona], aumentando a concentração e excreção destes nos fluidos corporais, evidenciando um quadro de cetonemia, cetonúria e cetoláctia, está associada com o aumento dos ácidos graxos não esterificados (AGNE) e esgotamento do glicogênio hepático e hipoglicemia (GONZÁLEZ; SILVA, 2006; BRUSS, 2008; NIELSEN e INGVARTSEN, 2004). 
O quadro clínico é evidenciado por hipofagia, queda na produção de leite, perda de peso e de escore de condição corporal (ECC), letargia, hiperexcitabilidade, hipercetonemia, hipoglicemia, hipoinsulinemia, altos níveis circulares de AGNE, aumento na concentração hepática de triglicerídeos, e baixo teor de glicogênio no interior dos hepatócitos (FONSECA et al., 2003; BRUSS, 2008). Na cetose subclínica são identificados cetonúria, cetonemia e cetoláctia, sem sinais clínicos evidentes (IWERSEN et al., 2009), estando presente queda na produção de leite e baixos níveis de glicose (FONSECA et al., 2003).

\section{Metabolismo Lipídico}

O tecido adiposo representa uma reserva corporal de energia, que consiste em células preenchidas com triglicerídeos, conhecidas como adipócitos. No interior destes, os triglicerídeos são continuamente quebrados e ressintetizados, resultando na libertação dos AGNE. Este processo é conhecido por lipólise e a síntese dos triglicerídeos é conhecida como lipogênese (KANEKO et al., 1997; HERDT, 2000).

Guard (2000) cita que animais em boas condições de saúde utilizam suas reservas corporais para obtenção de energia, contudo, há um limite para a quantidade de ácidos graxos que podem ser manipulados pelo organismo e utilizados pelo fígado. Segundo Grummer (1993) quando se atinge esse limite, os AGNE captados pelo fígado são oxidados ou esterificados em triglicerídeos e incorporados em lipoproteína de muito baixa densidade (LMBD) ou acumulados no tecido hepático.

Nos ruminantes, o tecido hepático possui baixa capacidade de exportar triacilglicerol na forma de LMBD, assim, os ácidos graxos são reesterificados e depositados na forma de triacilglicerol no fígado até que sejam eliminados, via oxidação completa ou cetogênese e uma pequena parte na forma de LMBD (SANTOS, 2006).

Na carência de glicose, os ácidos graxos também não são completamente oxidados, fazendo com que haja a formação de corpos cetônicos, os quais acabam se acumulando na circulação sanguínea (CHURCH,1993). O AcAc é o corpo cetônico inicialmente produzido durante a cetogênese hepática, este é espontaneamente descarboxilado em acetona e eliminada durante a eructação em ruminantes. A eliminação desta confere odor característico na expiração, com cheiro adocicado de acetona na cetose clínica (SANTOS, 2006).

\section{Métodos Preventivos}

O controle da cetose clínica está integralmente relacionado com a nutrição adequada da vaca durante o período seco e em lactação. Este engloba detalhes como consumo de matéria seca, 
digestibilidade da fibra, distribuição granulométrica, densidade de energia, teor de proteína, sistemas de alimentação e tamanho do rúmen (RADOSTITS et al., 2000).

As vacas não devem ser subalimentadas, nem ser gordas na época do parto. Uma frequência de alimentação extremamente baixa e a alimentação dos concentrados separada da forragem ao invés de uma ração completa pode levar a um aumento das taxas de cetose (MULLIGAN et al., 2006).

\section{Escore de condição corporal}

A prevenção da cetose requer manejo da condição corporal ao final da gestação, pois, animais obesos antes do parto tem um consumo de MS menor que animais com ECC adequado. O tecido adiposo afeta as concentrações de leptina que irá inibir o neuropeptídeo Y, este estimula o apetite por seu efeito nos centros da saciedade do sistema nervoso central. Deste modo, animais obesos podem ter concentrações mais altas de leptina ao final da gestação, inibindo indiretamente o apetite (SANTOS, 2006).

Corassin et al. (2011) relata que os animais super condicionados com ECC > 4,0 (escala de 1 a 5) são mais susceptíveis ao BEN após o parto devido à menor ingestão de MS, quando comparados a vacas com ECC entre 3,0 e 3,5, o qual é considerado adequado. Assim como quanto mais intenso for o BEN, maior será a mobilização de reservas lipídicas, proporcionando maior chance da ocorrência de cetose.

Os animais com maiores quantidades de gordura corpórea são predispostos a apresentar resistência à insulina, ocorrendo um aumento na mobilização de gordura e na quantidade de ácidos graxos livres no sangue. A maior concentração destes ácidos graxos no sangue inibe o consumo de MS pelos ruminantes (SANTOS, 2006).

\section{Manejo alimentar}

Logo após o parto a ingestão de MS aumenta dia após dia até chegar ao ponto máximo por volta da $10^{\mathrm{a}}$ a $12^{\mathrm{a}}$ semana de lactação, enquanto o pico de produção de leite ocorre por volta de 4 a 6 semanas pós-parto. Esta diferença na curva de produção de leite e ingestão de MS faz com que o animal enfrente, durante um período de \pm 60 dias, um desequilibro nutricional negativo, devido à mobilização de reserva corporal acumulada no final da lactação ou período seco (MATOS, 1995)

Vacas que recebem alimentação com excessiva densidade energética durante o período seco prolongado podem aumentar a predisposição ao desenvolvimento de cetose. Assim como os animais que recebem dietas com baixas quantidades de energia nas últimas semanas pré-parto, comprometem ainda mais a ingestão de energia já afetada pela queda natural do consumo de 
alimento que ocorre nos últimos dias de gestação e, acentuando o BEN. Portanto, animais sujeitos a restrição alimentar moderada por varias semanas antes do parto, apesar de apresentarem concentrações mais altas de ácidos graxos livres, têm menores concentrações hepáticas de triglicerídeos e lipídeos totais. (SANTOS, 2006).

Santos (2006) descreve também que dietas com baixas concentrações de fibra em detergente neutro $(\mathrm{FDN})(<35 \%)$ aumentam o consumo de MS pré-parto, porém, resulta em maior decréscimo de consumo na última semana de gestação. Já dietas com alta FDN (38\% a 45\%) ao final da gestação reduzem o consumo de MS no final da gestação, mas também minimizam a queda de ingestão de alimento na última semana de gestação. Dietas pré-parto devem ser formuladas para que vacas consumam os nutrientes necessários para a sua manutenção, crescimento fetal e síntese de colostro. Assim, dietas com nível moderado de FDN (35\% a 38\%) asseguram uma densidade energética adequada, e ao mesmo tempo evitam quedas abruptas em ingestão de MS.

\section{Ionóforos}

Os ionóforos são aditivos alimentares utilizados para incrementar a eficiência digestível em nível de rúmem, através de mudanças na fermentação, metabolismo, velocidade de passagem e população bacteriana (BORGES, 2003).

Eles alteram a flora bacteriana do rúmen, levando a uma diminuição das bactérias grampositivas, protozoários e fungos, além do aumento de bactérias gram-negativas. O efeito destas mudanças na flora bacteriana é o aumento da produção de propionato e diminuição na produção de acetato e butirato proporcionando mais precursores glucogênicos (PETERSSON-WOLFE et al., 2007; MARKANTONATOS et al., 2009).

A monensina sódica é a mais conhecida dos ionóforos, ela reduz a metanogênese ruminal, por divergir íons $\mathrm{C}$ e $\mathrm{H}+$ para outros produtos finais que não o metano. Esse redirecionamento leva à maior produção de propionato no rúmen, o que aumenta a densidade energética da dieta em 3 a 4\% e melhora a contribuição de glicose para o animal e resultam em redução da cetogênese em vacas leiteiras no início da lactação. Vacas que recebem 15 a $30 \mathrm{mg}$ de monensina por $\mathrm{kg}$ de dieta durante o final da gestação e inicio da lactação, possuem redução na incidência de cetose subclínica e clínica (SANTOS, 2006).

\section{Propilenoglicol}

O propilenoglicol alivia o BEN via ingestão forçada ou misturado na ração (STUDER et al., 1993). Além disso, previne a ocorrência de cetose devido as propriedades gliconeogênicas que modificam o metabolismo em vacas leiteiras (NIELSEN; INGVARTSEN, 2004; MIKULA 
et al., 2008). A utilização do propilenoglicol reduz as concentrações plasmáticas de BHB, ácidos graxos livres, uréia e aumentando as concentrações plasmáticas de glicose e insulina (GRUMMER et al., 1994; FORMIGONI et al., 1996).

De acordo com Santos (2006) quando incorporado na dieta, o propilenoglicol, pode reduzir o consumo de MS pelos animais, portanto, para que o seu uso seja efetivo, é necessário que seja fornecido por via oral, em quantidade de 300 a $500 \mathrm{~g}$ em uma dose única diária.

\section{Niacina}

A niacina atua no metabolismo de carboidratos, lipídeos, proteína, formação de ATP e regulação enzimática, desta forma a utilização de niacina na dieta pode prevenir a cetose, diminuindo a formação de ácidos graxos não esterificados, mantendo o nível de glicose no sangue e o consumo de MS (HUTJENS, 1992).

Durante a fermentação ruminal, os microrganismos sintetizam niacina e o aminoácido triptofano pode ser utilizado como precursor da síntese de niacina em células animais. Quando utilizada como aditivo alimentar, a sua função é a de reduzir a mobilização de gordura corporal durante o final da gestação e inicio da lactação. (SANTOS, 2006)

A niacina tem um efeito antilipolítico e induz aumento da glucose e insulina no sangue, esta deve ser fornecida a partir das duas semanas que antecedem o parto até a décima segunda semana após este (; RADOSTITS et al., 2000; HAYIRLI, 2006; GRUMMER, 2008), na quantidade de 6 a $12 \mathrm{~g} / \mathrm{dia}$ (SANTOS, 2006).

\section{CONCLUSÃO}

Com a intensificação da produção, a ocorrência de distúrbios metabólicos só tende a aumentar, por isso, é de grande relevância o conhecimento e a utilização de métodos preventivos para que estes possam ser evitados, bem como diminuir custos com tratamento e garantir sanidade, desempenho produtivo e reprodutivo dos animais. Durante o período de transição, é importante a realização de um manejo alimentar adequado aos animais, objetivando amenizar o balanço energético negativo, além da adequação do escorre de condição corporal, prevenindo assim a cetose. É relevante também a utilização de dietas aniônicas para prevenir desequilíbrios metabólicos como a hipocalcemia. 


\section{REFERÊNCIAS}

BEEDE, D. K. The DCAD concept: Transition rations for dry pregnant cows. Feeds tuffs. v.64, n.53, p.12, 1992.

BOBE, G.; YOUNG, J. W.; BEITZ, D. C. Invited Review: pathology, etiology, prevention, and treatment of fatty liver in dairy cows. Journal of Dairy Science, v.87, p. 3105-3124, 2004.

BORGES, F. B. Uso de niacina na alimentação de vacas de alta produção. Disponível em: <http:/www.zootec.com.br/niacina.htm>. Acesso em 25 abr. 2013.

BRUSS, L. M.; Lipids and ketones. In KANEKO, J. J.; HARVERY, W. J.; BRUSS, L. M. Clinical Biochemistry of Domestic Animals. 6. ed. San Diego, California, Academic Press, cap.4, p. 81-115, 2008. ISBN: 9780123704917.

CAVALIERI, F. L. B; SANTOS, G. T. dos. Balanço catiônico-aniônico em vacas leiteiras no pré-parto. Universidade Estadual de Maringá. 2000. Disponível em:

<http:/www.nupel.uem.br/balanço.pdf>. Acesso em: 22 abr. 2013.

CHURCH, D. C. Clasificación e importancia de los animales rumiantes. In: CHURCH, D. C. El Ruminante: fisiología digestiva y nutrición. Zaragoza: Acribia, cap. 1, p. 1-14, 1993.

CORASSIN, C. H.et al. Importância das desordens do periparto e seus fatores de risco sobre a produção de leite de vacas Holandesas. Semina: Ciências Agrárias, Londrina, v. 32, n. 3, p. 1101-1110, 2011.

CORBELLINI, C. N. Etiopatogênia e controle da hipocalcemia e hipomagnesemia em vacas leiteiras. Traduzido por Felix H. D. Gonzáles. In: GONZÁLES, F. H. D.; OSPINA, H. P.; BARCELLOS, J. O. J. (Eds.) In: SEMINÁRIO INTERNACIONAL SOBRE DEFICIÊNCIAS MINERAIS EM RUMINANTES. Anais... Porto Alegre: UFRGS, 1998.

CUNNINGHAM, J. G. Tratado de fisiologia dos animais domésticos. Rio de Janeiro: Guanabara Koogan, p. 710, 1993.

DAVIDSON, J. et al. Urine pH check helps avoid milk fever. Hoardís Dairyman, v.140, p.634, 1995.

DRACKLEY, J. K.et al. Metabolic Changes in Dairy Cows with Ketonemia in Response to Feed Restriction and Dietary 1,3-Butanedioll. Journal of Dairy Science, v. 75, p. 1622-1634, 1992.

DUKES, H. H. Fisiologia dos animais domésticos. 11 ed. Rio de Janeiro: Guanabara Koogan, p. 856, 1996.

FONSECA, L. F. L.et al. Suplementação de propilenoglicol para vacas no peri-parto: efeitos sobre incidência de cetose, produção leiteira, escore de condição corporal e primeiro estro pósparto. Acta Scientiarum Animal Sciences, v. 25, n. 1, p. 117-183, 2003.

FORMIGONI, A. et al. Effect of propylene glycol supplementation around parturition on milk yield, reproduction performance and some hormonal and metabolic characteristics in dairy cows. J. Dairy Res., Cambridge, v.63, n.1, p.11-24, 1996. 
GOFF, J. P.; HORST, R. L. Effect of addition of potassium and sodium, but not calcium, to prepartum rations on milk fever in dairy cows. Journal of Dairy Science. 80:176, 1997.

GOFF, J. P.; HORST, R. L. Physiological changues at parturition and their relationship to metabolic disord ers. Journal of Dairy Science, v. 80, p.1260-1268, 1997a.

GOFF, J. P. Pathophysiology of calcium and phosphorus disordens. Veterinary Clinics North America Food Animal Practice. V.16, p. 319 - 337, 2000.

GONZÁLEZ, F. H. D.; CAMPOS, R. Indicadores metabólico-nutricionais do leite. In: GONZÁleZ, F. H. D.; CAMPOS, R. (eds.): Anais do I Simpósio de Patologia Clínica Veterinária da Região Sul do Brasil. Porto Alegre: Gráfica da Universidade Federal do Rio Grande do Sul. p. 31-47, 2003.

GONZÁLEZ, F. H. D.; SILVA, S. C. Bioquímica clínica de lipídeos. Introdução á bioquímica clínica veterinária. 2. ed. Porto Alegre: Gráfica da Universidade Federal do Rio Grande do Sul. cap. 4, p. 213-226, 2006.

GRUMMER, R. R. et al. Effect of propylene glycol dosage during feed restriction on metabolites in blood of prepartum holstein heifers. Journal of Dairy Science, Savoy, v.77, n.12, p.36183623, 1994.

GRUMMER, R. R. Etiology of lipid-related metabolic disorders in periparturient dairy cows. Journal of Dairy Science, v.76, p.3882-3896, 1993.

GRUMMER, R. R. Nutritional and management strategies for the prevention of fatty liver in dairy cattle. The Veterinary Journal, v. 176, p.10-20, 2008.

GUARD, C. Doenças Metabólicas: Uma abordagem de rebanho. In: Rebhun, W. C. Doenças do Gado Leiteiro. São Paulo: Rocca, (15): 613-620, 2000.

HAYIRLI, A.et al. Animal and dietary factors effecting feed intake during the prefesh transition period in Holsteins. Journal of dairy Science, v. 85, n. 12, p. 3430-3443, 2002.

HAYIRLI, A. The Role of Exogenous Insulin in the Complex of Hepatic Lipidosis and Ketosis Associated with Insulin Resistance Phenomenon in Postpartum Dairy Cattle. Veterinary

Research Communications, 30(7), 749-774, 2006.

HERDT, T. H. Ruminant adaptation to negative energy balance. Influences on the etiology of ketosis and fatty liver. The Veterinary Clinics of North America. Food Animal Practice, 16(2), 215-230, 2000.

HORST, R. L.et al. Strategies for preventing milk fever in dairy cattle. Journal of dairy science, v. 80, n. 7, p. 80:1269, 1997.

HUTJENS, M.F. Selecting feed additives. In VAN HORN, H.H., WILCOX, C.J. Large dairy herd management.Champaign, EUA, p.309-317, 1992.

IWERSEN, M.et al. Evaluation of an electronic cowside test to detect subclinical ketosis in dairy cows. Journal of Dairy Sience, v. 92, p. 2618 - 2624, 2009.

JONES, T. C.; HUNT, R. D.; KING, N. W. Patologia veterinaria. 6 ed. São Paulo: Manole, p. 813-815, 2000. 
JUCHEM, S. O.et al. Production and blood parameter of holstein cows treated prepartum with sodium monensin or propylene glycol. Journal of Dairy Science, v.87, p.680-689, 2004.

KANEKO, J. J., HARVEY, J. W.; BRUSS, M. Clinical biochemistry of domestic animals, Gulf Professional Publishing, 1997.

KUNKEL, A. J. Our most costly dairy cow disease... 25 years later. Hoard's Dairyman p.788, 2007.

LEAN, I. J.; DEGARIS, P. J. Milk fever in Dairy cows: A review of pathophysiology and control principles. The Veterinary Journal, v. 176, n. 1, p. 58-69, 2009.

LE BLANC, S. J., LESLIE, K. E., DUFFIELD, T. F. Metabolic predictors of displaced abomasums in dairy cattle. Journal of Dairy Science, 88, 159-170, 2005.

MARKANTONATOS, X. et al. Effects of monensin on volatile fatty acid metabolism in periparturient dairy cows using compartmental analysis. Animal Feed Science and Technology, 153(1-2), 11-27, 2009

MATOS, L.L. Perspectivas em alimentação e manejo de vacas em lactação. In: ANAIS DA XXXII REUNIÃO ANUAL DA SBZ. Anais..., Brasília - DF, p.147-155, 1995.

MIKULA, R.et al. Effects of propylene glycol supplementation on blood biochemical parameters in dairy cows. Bull. Vet. Inst. Polawy, v. 52, p. 461-466, 2008.

MOORE, S.J.; VANDEHAAR, M.J.; SHARMA, B.K. et al. Effects of altering dietary cationanion difference on calcium and energy metabolism in peripartum cows. Journal of Dairy Science, v.83, n.9, p.2095-2104, 2000.

MULLIGAN, F.et al. A herd health approach to dairy cow nutrition and production diseases of the transition cow. Animal Reproduction Science, vol. 96, 331-353, 2006.

NAGARAJA, T. G.; TITGEMEYER, E. C. Ruminal acidosis in beef cattle: the current microbiological and nutritional outlook. Journal of Dairy Science, Champaign, v. 90, sup., p. E17-E38, 2007.

NETO, A. C.et al. Problemas metabólicos provenientes do manejo nutricional incorreto em vacas leiteiras de alta produção recém paridas. Revista eletrônica de veterinária, v.12, n. 11, 2011.

NIELSEN, N. I.; INGVARTSEN, K. L. propylene glycol for dairy cows A review of the metabolism of propylene glycol and its effects on physiological parameters, feed intake, milk production and risk of ketosis. Animal Feed Science and Technology, v. 115, p. 191-213, 2004.

NRC. Nutrient Requirements of Dairy Cattle. $7^{\text {th }}$ edition. National Academy Press. Washington, D.C. 2001.

OBAA, M.; OAKLEY, A.; TREMBLAY, G. Dietary Ca concentration to minimize the risk of hypocalcaemia in dairy cows is affected by the dietary cation-anion difference. Animal Feed Science and Technology, vol. 164, 147-153, 2011.

OETZEL, G. R. Improving reproductive performance in dairy cattle via milk fever prevention. In Proc. $28^{\text {th }}$ Annual Convention Am. Assoc. Bov. Pract. San Antonio, TX. Pag. 52, 1995. 
PETERSSON-WOLFE, C. et al. Effect of Monensin Delivery Method on Dry Matter Intake, Body Condition Score, and Metabolic Parameters in Transition Dairy Cows. Journal of Dairy Science, 90(4), 1870-1879, 2007.

RADOSTITS, O. M., ARUNDEL, J. H.; GAY, C. C. Veterinary medicine, Elsevier Health Sciences. 2000.

RADOSTITS, O. M. Clínica veterinária: um tratado de doenças de bovinos, ovinos, suínos, caprinos e equinos. 9 ed. Rio de Janeiro: Guanabara Koogan, p. 1278 - 1291, 2002.

RAMOS-NIEVES, J.et al. Effects of anion supplementation to low-potassium prepartum diets on macromineral status and performance of periparturient dairy cows. Journal of Dairy Science, vol. 92, n. 11, 5677-5691, 2009.

RÉRAT, M.et al. Effect of different potassium levels in hay on acid-base status and mineral balance in periparturient dairy cows. Journal of Dairy Science, vol. 92, n. 12, 6123-6133, 2009.

RODRIGUES, R. Distúrbios do metabolismo de cálcio: hipocalcemia puerperal e Eclampsia. Porto Alegre: Universidade Federal do Rio Grande do Sul, p. 2-11, 2004. Disponível em: <http:/www.ufrgs.br/favet/registro/pdf/disturbios_calcio.pdf>. Acesso em: 03 de out. 2011.

RUKKWAMSUK, T.et al. Effect of propylene glycol on fatty liver development and hepatic fructose 1,6 biphosphatase activity in periparturient dairy cows. Livestock Production Science, v. 95, p. 95-102, 2005.

SANTOS, J. E. P. Distúrbios metabólicos. In: BERCHIELI, T. T.; PIRES, A. V.; OLIVEIRA, S. G. Nutrição de ruminantes. FUNEP. Jaboticabal. 2006.

STUDER, V. A. et al. Effect of prepartum propylene glycol administration on periparturient fatty liver in dairy cows. Journal of Dairy Science, v.76, n.10, p.2931-2939, 1993.

TEDESCO, D.et al. Effects of silymarin, a natural hepatoprotetor, in periparturient dairy cows. Journal of Dairy Science, v.87, p. 2239-2247, 2004.

TEIXEIRA, L. F. A. C.et al. Efeito do estradiol, dietas e duração do período seco sobre o consumo de matéria seca de vacas holandesas. Ciência e agrotecnologia. v. 27, n. 4, p. 903-911, 2003.

THILSING-HANSEN, T.; JORGENSEN, R.; OSTERGAARD, S. Milk Fever Control Principles: A Review. Acta Veterinary Scandinavica, vol. 43, 1-19, 2002.

WHATHES, D. C.et al Differences between primiparous and multiparous dairy cows in the interrelationsships between metabolic traits, milk yield and body condition score in the periparturient period. Domestic Animal Endocrinology, v. 33, p. 203-225, 2007.

VAN KNEGSEL, A. T. M.et al. Effect on glucogenic vs. Lipogenic diets on energy balance, blood metabolites, and reproduction in primiparus and multiparous dairy cows in early lactation. Journal of Dairy Science, v. 90, p.3397-3409, 2007. 
